\title{
A FLEXIBLE LOCATION-CONTEXT REPRESENTATION
}

\author{
Filipe Meneses, Adriano Moreira \\ Information Systems Department, University of Minho, Campus Azurém, 4800-058 Guimarães, Portugal, \\ \{meneses, adriano\}@dsi.uminho.pt
}

\begin{abstract}
Ubiquitous computing and the development of context-aware applications have been limited by the lack of open and generic solutions. In this paper we propose a flexible location-context representation which supports data acquired through multiple sensors represented in different space models.
\end{abstract}

Keywords - Context Representation, Context Management, Location Management.

\section{INTRODUCTION}

The technological evolution achieved during the lasts years promoted a pervasive environment with more computing devices, wireless networks and more sophisticated sensors.

The exploitation of these new and rich environments for ubiquitous computing requires the development of open, scalable and flexible solutions for multiple problems, including context management. Among these, the necessity to evolve the notion of context is being seen as one of the most crucial.

During these last years, many context-aware and locationbased services systems were developed. However, many of the results were achieved through simple implementations where location and other contextual data were used directly from sensors to provide adaptation or selection of information accordingly to the user context.

In this paper we propose an innovative representation for the location context of a mobile user by aggregating contextual information in a multidimensional space. The proposed approach is based on an open and generic entity capable of supporting virtually any sensor or location service without impose any specific space model. Moreover, it is capable of supporting data globally useful, at the same time that holds data linked to local or specific space models.

The remainder of this paper is organized as follows. In section 2 we review the work done by other authors. In sections 3 we present our notion of position, location and context. In section 4 and 5 we present why is important have a generic solution capable of supporting any space model and show the proposed solution to context representation. In the section 6 we present a solution for the context management and how it is being evaluated. Finally, in section 7 , we present our conclusions and future work.

\section{RELATED WORK}

Active Badge was one of the first technologies developed to acquire the location of a user inside an office. Location was expressed in a symbolic way, such a room or the area around a desk [1].

Leonhardt [2], [3] extended the concept of location service by considering that multiple sensors can be used to simultaneously track the position of objects, and that sensors and location-aware applications can reside on different nodes in an open distributed system. In a scenario of a hierarchical location model, Leonhardt proposed an acquisition function to integrate the data collected from multiple sensors. This function provided an abstraction layer that made the location information available to applications as one piece of information and allowed to develop applications independently from the sensors technologies.

Leonhardi also addressed the idea of a universal location service [4]. He proposed an architecture for a distributed location service based on the Internet. Through a location server, the clients could ask for the objects at a given location or for the location of a mobile object. This system was based on a topological model instead of a symbolic model, using the WGS84 datum.

The Location Inter-operability Forum (LIF) [5] is also specifying a location service for cellular radio networks. The Mobile Location Protocol (MLP) provides applications with the possibility to query a server for the location of mobile stations independent of the underlying network technology. The specification foresees several types of location services. Among them, it includes the Standard Location Immediate Service that returns the actual position of a station and the Triggered Location Reporting Service that notifies a Location Services Client at a specific time interval or on the occurrence of a specific event.

These and other known location systems provide a uniform representation of location that is independent of the positioning technologies. It simplifies the development of context-aware applications, providing applications with a common query language to access location information.

The Context Toolkit approach gave a step further in the issue of context representation [6]. Here, a network of heterogeneous sensors that expose their functionality through a normalized network interface - the widgets, replaced the idea of a homogeneous location service located 
on the infrastructure. This approach also provides support for many different types of context sources.

Jason el al [7] proposed an approach where most the acquisition, processing and storage of contextual data is done in the infrastructure. In order to reduce the capabilities required from the mobile devices, the applications could also run on the infrastructure.

In the same direction, Judd et al. [8] proposed a solution based on a limited number of service classes and a Contextual Service Interface to access contextual information using a set of pre-defined query types. However, the discovery of the network services by applications is not addressed, as well as security and privacy.

Judd et al. approach identifies the necessity to define a data format as a key requirement to implement the proposed solution. Similarly, Jason et al. describe the definition of a standardized communication protocol and the definition of the data formats required for applications to access contextual data as the two major research challenges that have to be addressed before a context-aware infrastructure approach can be deployed successfully.

Some of the known systems represent the space as symbolic locations (almost always in hierarchical structures) while others use geographic coordinates. Jiang [9] proposes a hybrid system where a URN is used to express the location, combining the facilities of the geographic systems with the convenience of the symbol location.

Hazas et al [10] argues that is necessary to fusion the data from multiple sensors and there is not a single representation of location data that is useful in all circumstances. Often the applications need high-level contextual information that should be produced based on the location data.

\section{CONCEPTS}

The terms position, location and context are often used to describe the same, or similar, data. In particular, position and location are often being used to represent "where a user is". Here we define position and location with two different meanings.

A large number of technologies and mechanisms can be used to retrieve the position of a mobile user or device. For example, a GPS receiver can acquire the geographic position of a mobile user as a pair of geographic coordinates. In the Active Badge System [1], position is represented by the code associated to a certain infrared sensor. Position can also be represented in absolute terms, such as a pair of geographic coordinates in a well known referential (e.g. WGS84), or in symbolic terms, such as the cellID in a mobile cellular network.

Space models are a representation of a certain geographic area and are maintained by any administrative entity.
Examples of space models are: a) the geographic division of the space as a set of polygons defined by a list of geographic points, such as the borders of countries; b) the geographic position of the centre of a cell in a cellular network, referred by the cellID, and the corresponding cell radius; c) the list of rooms covered by a certain WLAN Access Point, referred by the identification of the Access Point; d) the meaning of a list of codes sensed through a Bluetooth interface. This concept of space model is the enabler to support multiple representations of the same geographic region, as well as to support space representations maintained by multiple independent administrative domains.

The position information (raw data) is seen as location information when used on top of a Space Model. For example, a pair of geographic coordinates (position) may represent a certain street (location) if represented on top of a streets map (space model). Similarly, when we use an Active Badge we have our location if we have a referential (space model) that tells us where in the building is located the infrared sensor defining a cell that detected our badge. The existence of a space model enables the transformation of position information into location information - although at the same position, different users can be at different locations, if different space models are used in the transformation. The opposite can also be true: two different users at different positions may be in the same location (e.g. the same town).

Space models can also support the translation between different formats for position/location representation. This is an alternative approach to the path creation suggested in [11] for translation between, for example, a GPS point and a ZIP code.

Location describes better the context of a user than his position. However, position and location are impersonal until they are bound to a person or object. Again, two different users can be in different contexts while standing at the same location: "I am at home while my friend is at a friend's home".

We define context as all the information that characterizes the user in a specific moment. Although location has been the more exploited dimension of a user context, many other dimensions can be used to support the adaptation of contextaware applications. A context may include dimensions such as the user position, expressed in many different referentials, the user location, the user activity, a list of nearby objects (or people), the available resources, etc.

The value of some basic dimensions of a context may be obtained directly from physical sensors (position, orientation, ambient light level, room temperature, etc.). Other dimensions may be calculated from raw data (speed, acceleration, location, list of nearby objects, etc.). Some other dimensions may even be estimated from the information provided by other dimensions (activity may be 
estimated from position history and time, more precise location from position and street maps, etc.).

In this paper, we concentrate our discussion mainly on the position/location dimensions of a user's context - the location context.

\section{SPACE MODELS}

As seen in section II, several authors have shown successful cases of systems where position and location information is used to build context-aware applications and location-based services. However, all the known solutions imposed some constrains: use of specific sensors, use the sensed data in a specific format, etc.

A major issue in context-aware computing is the design of data formats used to exchange contextual information. Along with the set of protocols required to transfer contextual data, the data formats are one of the research challenges for ubiquitous computing [7]. Most of the reported approaches to context-aware systems use proprietary data formats to describe context, in particular in what concerns position and location. This has lead to prototypes that demonstrate context-aware systems that work only within the lab or within very restricted and controlled environments.

On the other hand, the context of a user is a point in a multidimensional space with an unbounded number of dimensions. Even for location only, we cannot predict all the sensors that will be available in the future or predict the needs of applications in terms of contextual information. We should then consider a context representation that is, simultaneously, usable by applications, independent from the context sources, and also flexible enough to accommodate future needs.

The majority of the systems are supported by topological or hierarchical space models. Topological space models represent the environment in a grid and every place can be referenced by a par of coordinates. The GPS coordinate system is a well know world wide example of a coordinate system. Hierarchical space models represent the physical environment in different levels (hierarchical relationships). An example of a hierarchical model is the address of a place where a building belongs to a street; the street is contained in a city, etc.

Both models have advantages and disadvantages that often make one of them more suitable for a specific case. In [9], Jiang et al. proposes a hybrid model, that combines the hierarchical and coordinate systems.

Other space models can be used to specific situations or in restricted domains. For example a museum may place numerical place tags near each painting. Although the tags associated to each painting are not expressed in a hierarchical or coordinate space model, they can be useful for specific application to retrieve more information about the viewed painting.

Local applications are those who scope is restricted to a certain location or area, such a specialized application to guide the user inside a museum or airport. Frequently, local applications rely on local contextual data, using local specific space models.

Global applications are those that, although location or context-aware, are useful everywhere or, at least, can be used in many different scenarios. Theses applications tend to be dependent on global space models.

\section{CONTEXT REPRESENTATION}

Taking these issues into account, we propose a data format for context representation based on the following principles:

\section{A. Unbound and dynamic list of attributes}

The context of a user (or other entity) is an unbounded and dynamic list of attributes, represented by standardized and non-standardized data structures. It is unbounded because there is no limit on the number and nature of the used attributes. It is dynamic because the list of attributes can vary with time and because the values of those attributes are also time variant.

\section{B. Cumulative storage of knowledge}

The context is a cumulative storage of knowledge about the user history, being able to remember past experiences and situations. As an example, the context must be aware that a user visiting a certain town has already been there some time ago.

\section{Mandatory and optional attributes}

The context is described by a fixed number of mandatory attributes and a variable number of optional attributes.

The mandatory attributes have well defined data formats (e.g. the context must always include the geographic position of the user, described as a pair of geographic coordinates - latitude, longitude pair - in the WGS84 datum). Whenever the value of a mandatory dimension is unavailable, it must be set to "unknown", as also suggested in [7]. The set of mandatory attributes are used to represent the few most relevant and widely used context dimensions.

The variable number of optional attributes, described by an XML stream, have an arbitrary data format. Although optional, the representation of these attributes may also be used through normalized data formats. Here, the term optional means that they are not required to be available in a context representation. However, one expects that most of the attributes in a context representation will be optional. 


\section{Three different categories}

The context attributes are classified into the following categories: primary, calculated or estimated.

Primary attributes represent raw data acquired directly from sensors (e.g. the cellID code in a cellular network), or data retrieved from space models (e.g. the cellID can be used as a key to retrieve the geographic coordinates of the centre of the cell and its radius from a space model server).

Calculated attributes represent contextual data that can be calculated from raw data (e.g. the speed of a user can be calculated from successive position readings). These attributes can be calculated by a context manager on its own or with the help of network services.

Estimated attributes represent contextual data that can be estimated from other attributes. For example, the context manager can estimate the name of the street a user it at by querying a maps server with the geographic coordinates of the user; the result would be: the user is at the Liberty Street, with a probability of $83 \%$.

When an arbitrary context-aware application retrieves the context of a certain user, it must be able to understand and process mandatory attributes. On the other hand, it may, or may not, understand all or a part of the optional attributes.

We propose the following set of mandatory attributes:

- Position - the user position expressed by the city and country names;

- Geographic position - a par of coordinates and the used datum;

- Time zone - the time zone of the place where the user is located (eg.: GMT+1);

- Timestamp - a timestamp of the last change occurred in any of the user context dimensions.

\section{CONTEXT MANAGEMENT}

We developed a Context Manager system that supports a Context Object with all the characteristics identified in the previous section. The system collects the contextual data, processes it and provides an interface for applications to access contextual data. The system integrates contextual data collected from the user devices and raw data from local context sources. In figure 1 we show the major components that interact with the Context Manager.

The positioning data is received by the Context Manager Feeder Interface which is implemented in three different technologies: SOAP, UDP and RMI. This triple implementation allows the Context Handler applications (that run near the sensors and are responsible to the transmission of the data) to transmit their data using the technology that is most convenient to them. Location Services that exist on the network also uses this interface to transmit the user position.

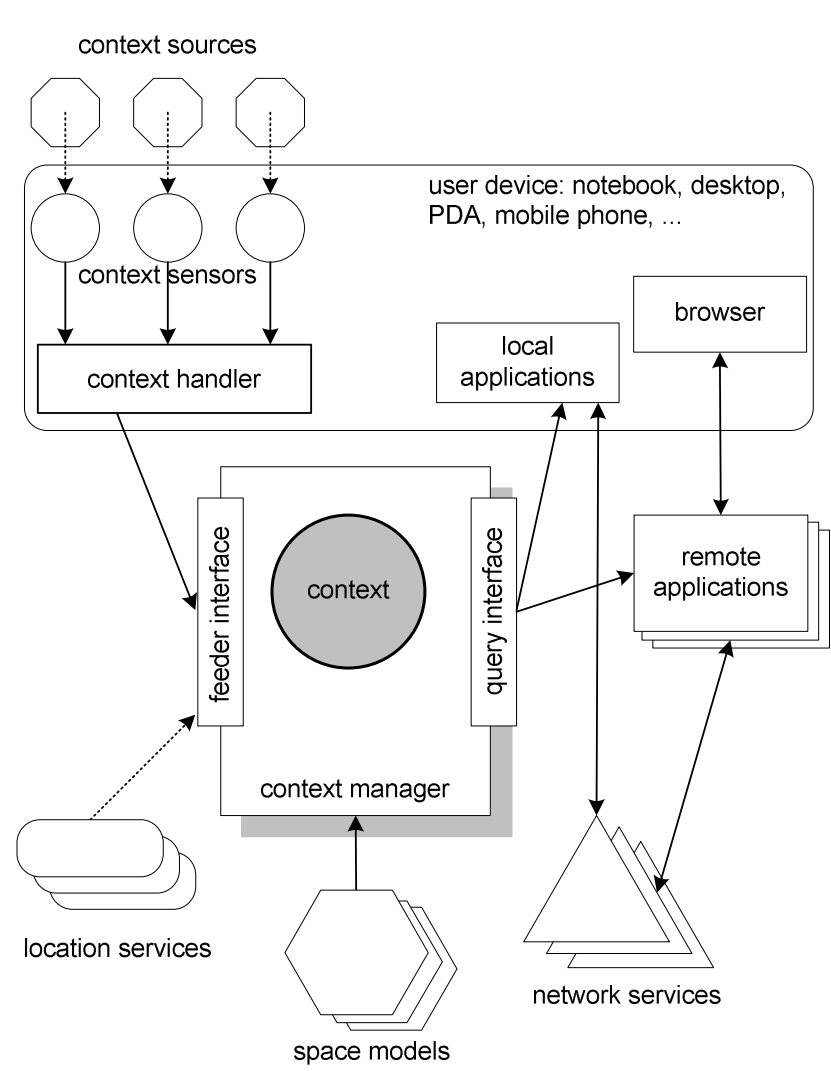

For each raw data received, the Context Manager queries a Space Model server seeking for more information about the received data. The Space Model servers answer is a XML stream.

The applications that use the contextual information act as client of the Context Manager service. The client applications use the Query Interface to request user contextual information or to request to be notified when changes occur in the user context (in any dimension or in a specific dimension). The applications who request this kind of notifications must implement the Context Notification Interface that will be used by the Context Manager system to do the notifications. The access to the Context Manager through the feeder and query interfaces is controlled by a password. The privacy of the users is controlled by each user by providing a username and a password only to the set of trusted applications.

The system is being evaluated in the VADE project [12]. The VADE project explores the concept of Value ADded Environment (VADE) as a new model for the selection and use of location-based services. The users interact with the system through a portal using a PDA, cellular phone or notebook computers. The user context is used by some global applications and services to produce content based on the user location. The portal can also integrate local applications that can use the user context representation to produce fine-grained content. 
Using a centralized system to manage the user context ended to be adequate, allowing the applications to abstract to interact with the sensors and locations systems. In this prototype we are using the following context representation:

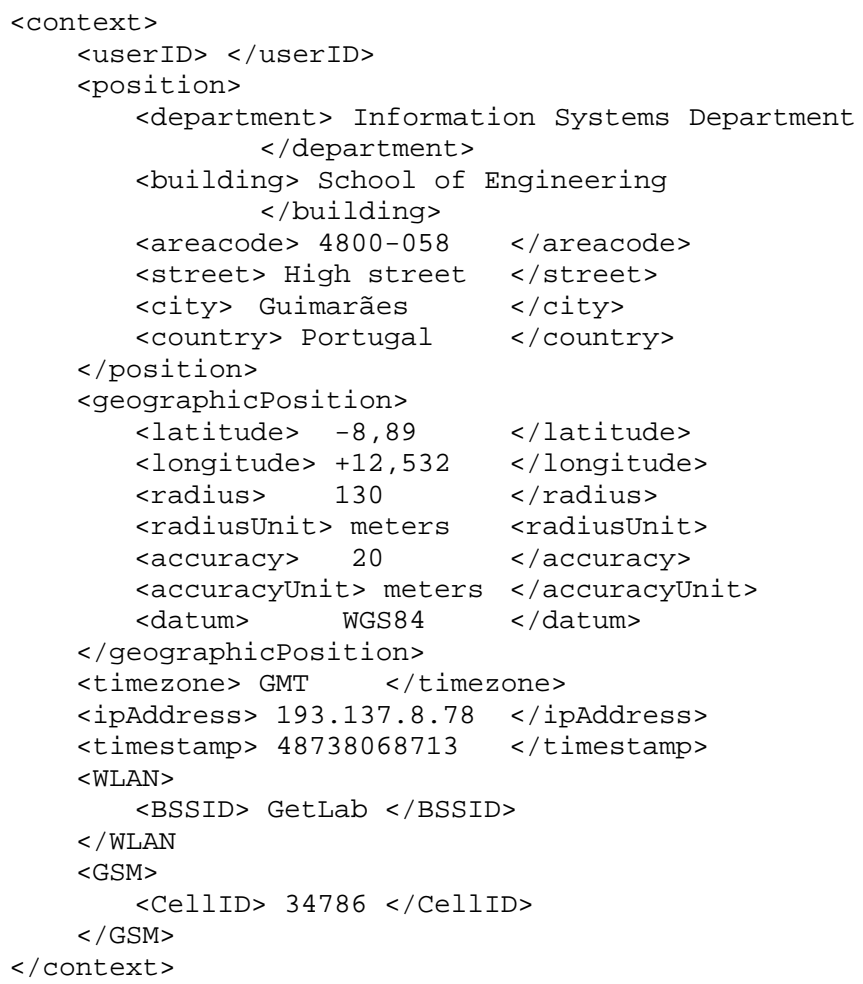

We are using XML in our prototype implementation because it simplifies the exchange of contextual data between the system components. Other representations however are possible.

This context representation shown to be appropriated to the different kind of applications developed. It fulfilled the needs of global applications and, simultaneously, covered the requirements of different specialized local applications.

\section{CONCLUSIONS}

The literature shows that in most of the cases no attention is given to the contextual data representation. Our solution, with a set of normalized fields, allows the development of applications without being dependent on the sensors used to acquire the contextual data.

Our generic representation supports the addition of new data without imposing any space model. This leads to a generic solution capable of dealing with new sensors and contextual data. Supporting any space models, the architecture grants the existence of contextual data with meaning only to a specific place as it supports global data.

We are now further developing the context representation by adding to the Context Manager the capability to add calculated and estimated attributes. We expect to identify known or familiar places/locations (eg. the user is at home) as it can be very attractive to some applications.

\section{AKNOWLEDGEMENTS}

This work was supported by the Portuguese Foundation for Science and Technology (FCT) (grant number SFRH/BD/8279/2002)

\section{REFERENCES}

[1] A. Harter and A. Hopper, "A distributed location system for the active office", IEEE Network, vol. 8, pp. 62-70, 1994.

[2] U. Leonhardt, "Supporting Location-awareness in Open Distributed Systems". PhD thesis, Department of Computing, Imperial College, London, 1998.

[3] U. Leonhardt and J. Magee, "Multi-sensor Location Tracking", in Proceedings of 4th ACM/IEEE Internation Conference on Mobile Computing and Networking - MOBICOM98, 1998.

[4] A. Leonhardi and K. Rothermel, "A Comparison of Protocols for Updating Location Information". Technical Report. University of Stuttgart, 2000.

[5] LIF Location Interoperability Forum, website, available as "http://www.openmobilealliance.org/lif/".

[6] D. Salber, A. K. Dey, and G. D. Abowd, "The Context Toolkit: Aiding the development of context-enabled applications", in Proceedings of 1999 Conference on Human Factors in Computing Systems (CHI'99), 1999.

[7] J. I. Hong and J. A. Landay, "An Infrastructure Approach to Context-Aware Computing", HumanComputer Interaction Journal, vol. 16, pp. 2-3, 2001.

[8] G. Judd and P. Steenkiste, "Providing Contextual Information to Ubiquitous Computing Applications", in Proceedings of First IEEE International Conference on Pervasive Computing and Communications (PerCom'03), Dallas-Fort Worth, Texas, 2003.

[9] C. Jiang and P. Steenkiste, "A Hybrid Location Model with a Computable Location Identifier for Ubiquitous Computing", in Proceedings of The Fourth International Conference on Ubiquitous Computing (UBICOMP 2002), Goteborg, Sweden, 2002.

[10] M. Hazas, J. Scott, and J. Krumm, "Location-aware Computing Comes of Age", Computer, vol. 37, pp. 9597, February 2004.

[11]E. Kiciman and A. Fox, "Using Dynamic Mediation to Integrate COTS Entities in a Ubiquitous Computing Environment", in Proceedings of 2nd International Symposium on Handheld and Ubiquitous Computing (HUC2K), Bristol, UK, 2000.

[12] Value Added Environments for Dynamic Support to Location-based Services in UMTS Networks, website, available as "http://get.dsi.uminho.pt/vade". 